\title{
Engineering implant surfaces by hyalưronan to control peri implant tissue response: in vitro, animal and clinical testing
}

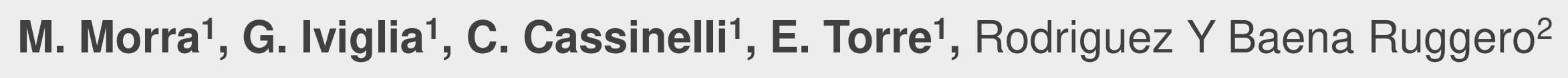

${ }^{1}$ Nobil Bio Ricerche srl, Via Valcastellana 26, 14037 Portacomaro, Italy

2Dept. Clinico-Surgical, Diagnostic and Pediatric Sciences, School of Dentistry, University of Pavia, Pavia, Italy

\section{Abstract}

Presently, oral implantology controls the fixture host tissue interaction by surface topography. The prevalence of peri implantitis on the long term suggests that implant surfaces should not just promote osteointegration, but also moderate periimplant inflammatory response. A possible approach involves enabling implant surfaces to guide peri implant tissue healing through biological molecules linked to the surface. This communication presents in vitro, animal and clinical results on titanium implants bearing surface-linked nanolayers of hyaluronan, a main inflammation control and tissue repair.
player in

Hyaluronan, $\mathrm{HA}$
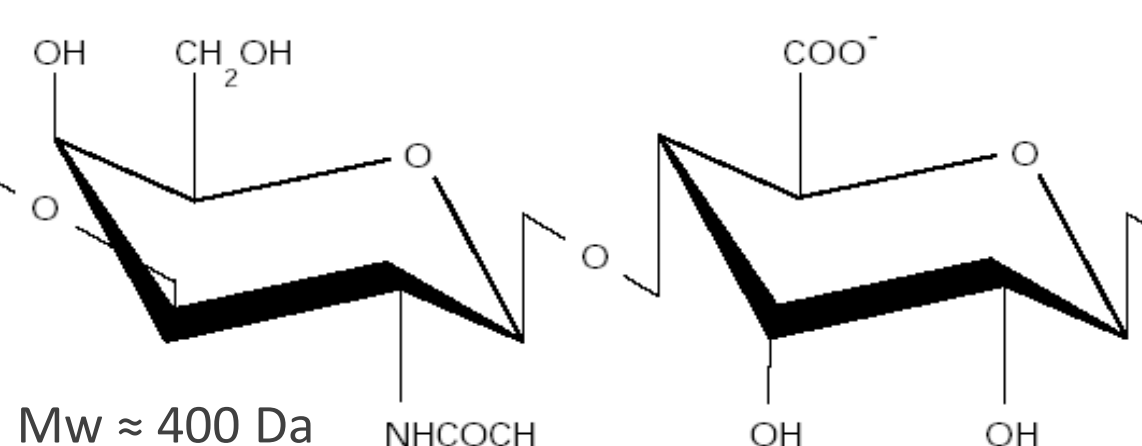

$\mathrm{N}$-acetyl-D-glucosamine - D-glucuronate linked by $\beta 1-4$ and $\beta 1-3$ linkages

It is present in all tissues

\section{Background and Aim}

Based on our background on surface modification of implant surfaces by covalent linking of biological molecules, the aim of this work was to test at different levels the following hypothesis:

hyaluronan, the main glycosaminoglican mammalians use in tissue repair, can affect implant/host tissue response. In particular, it affects the balance between inflammatory stimuli from bacterial aggression and response of defence systems, the main reason for peri implant tissue destruction.

Surface modfication of titanium implant by hyaluronan presents to the host tissue the antiinflammatory and proosteogenic hyalurona chemistry

Titanium implants surface-modifiied by hyaluronan should provide enhanced bone regenration and antiinflammatory properties

\section{Methods and Materials}

Medium Mw HA $(800 \mathrm{kDa})$ from bacterial fermentation is covalently linked to the titanium surface through an aminated layer

Amino-groups rich surface

Ti IMPLANT

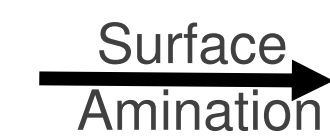

Chemical coupling

(carboxyl + surface amino)

\section{HA-coated \\ surface}

Surface characterization was conducted by X-ray Photoelectron Spectroscopy (XPS) analysis, SEM-EDX, $\zeta$ potential measurement.

The expression of inflammation-related genes in vitro, using J774A.1 macrophages, after stimulation of inflammatory response by Lypopolysaccharide or free-radical release was evaluated by RT-PCR measurement.

Bone regeneration was measured in a rabbit model through histomorphometry at 4 weeks after implantation.
- The clinical trial: "Blind comparison of covalently-linked hyaluronan VS controldental implants in a randomized crossove clinical investigation" was conducted at the University of Pavia Ity principal University Principal investigator Prof. Rodriguez Y Baena according to the ISO 14155-11, and GCP. A total of 106 implants in 29 subjects were loaded and were taken as baseline for RX values (mesial and distal), to 1 year, plus a 2 years follow-up

\section{Results}

Surface chemical analysis by XPS confirms that the chemistry of the titanium implant surface turns into the hyaluronan chemistry: Ti disappears, the $C$ peak grows and the $\mathrm{N}$ peak appears. The uniformity of the surface HA-layer is confirmed by the linearity and smoothness of the curve between 900 and $600 \mathrm{eV}$.

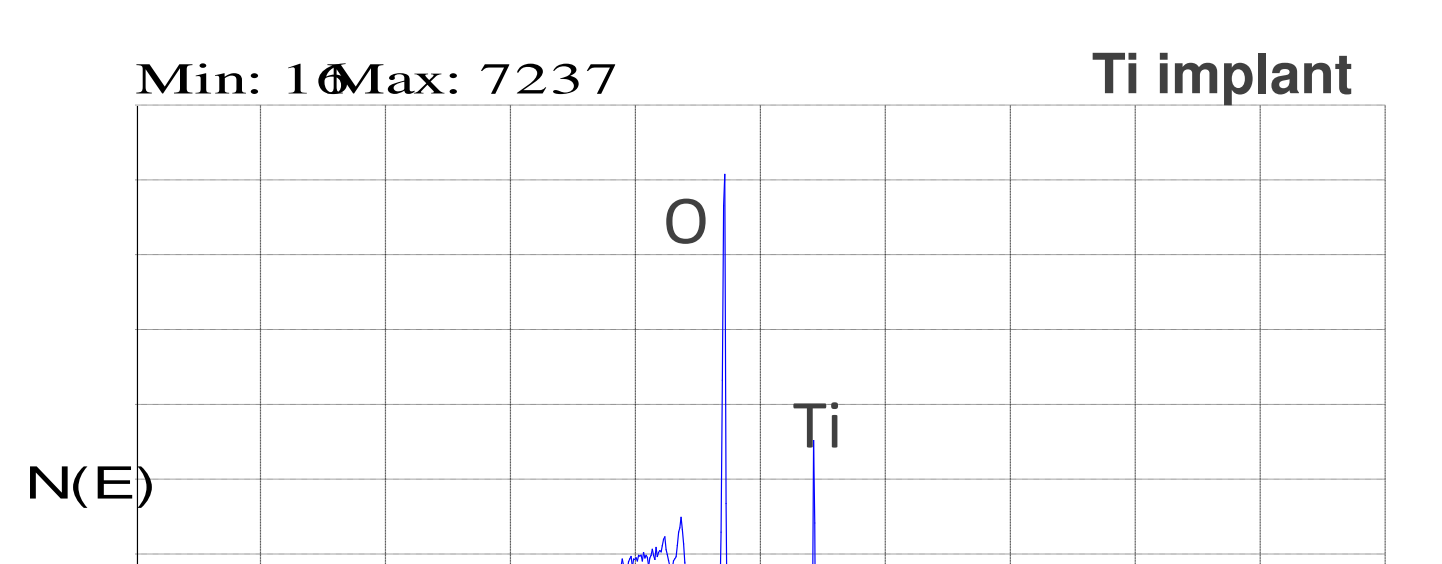

1000900800700600500400300200100 Binding Energy (eV)

Min: 1 Max: 21807 HA-coated Ti implant

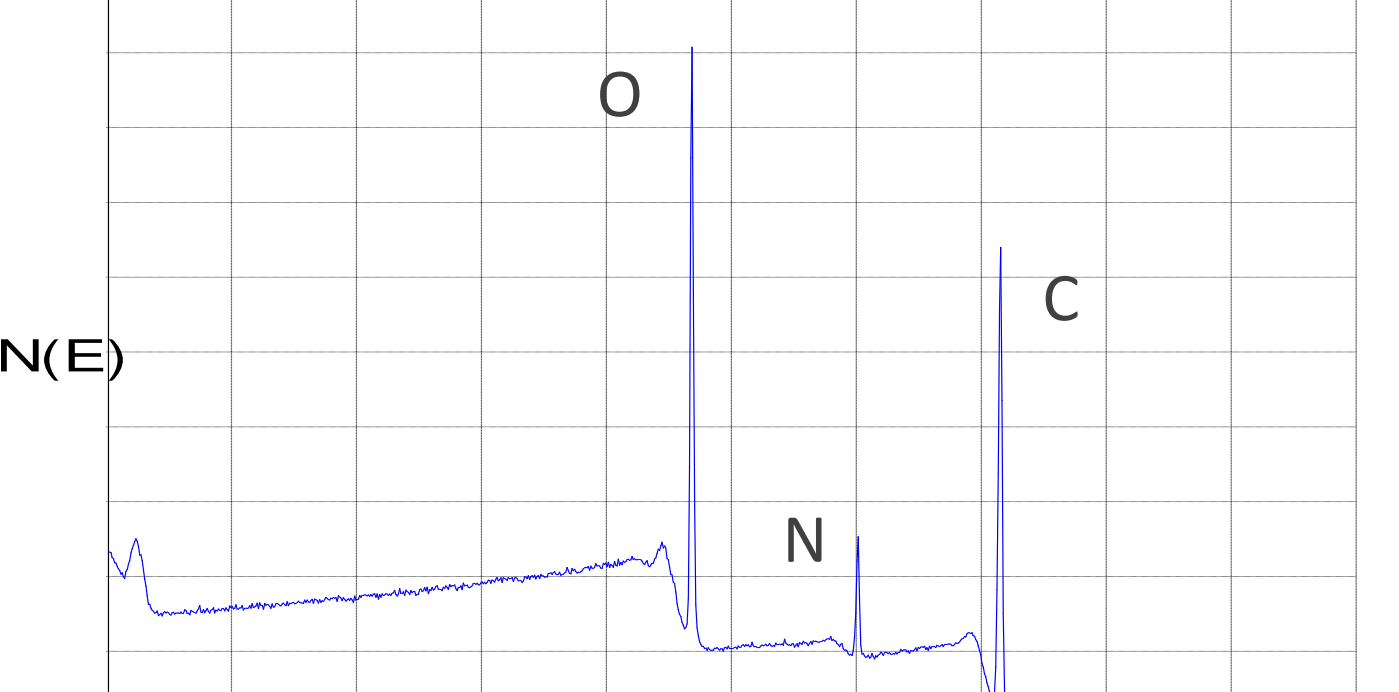
1000900800700600500400300200100
Binding Energy $(\mathrm{eV})$

Surface $\zeta$ - potential analysis strengthens the results obtained with XPS. Since Ti has an intrinsic material conductance which influence the streaming potential measurements, a streaming current was recorded. A native $\mathrm{TiO}_{2}$ layer covering the surface introduces an amphoteric surface with an IEP around 3.7, slightly lower than what is found in literature $(4.4-4.2)$, probably due to the surface roughness obtained with the in-house double acid etching and a glow discharge treatment before the analysis.

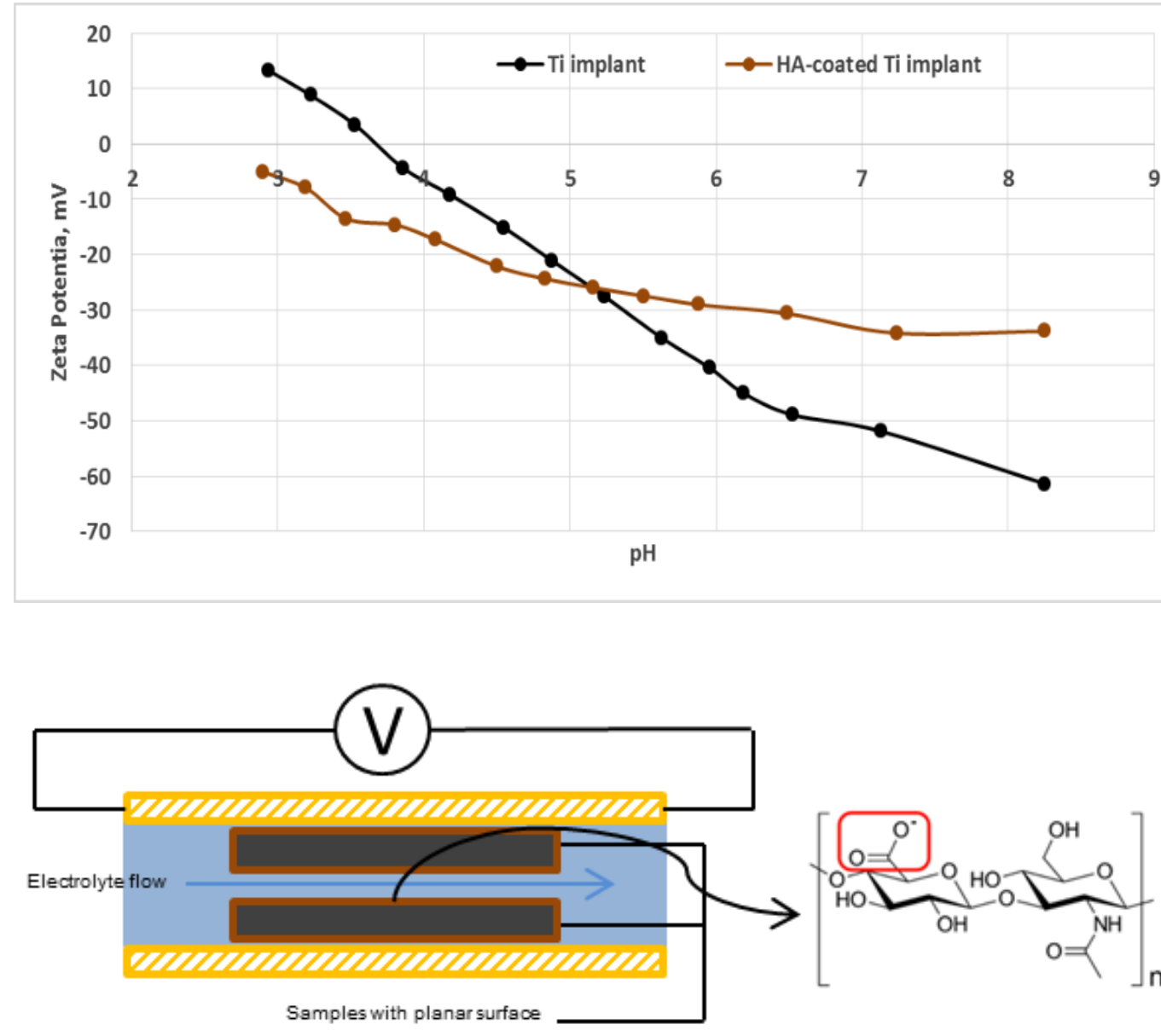

Hyaluronic acid, in solution shows an acidic behavior with a pKa around 2.9. Streaming current behavior with a pKa around 2.9. Streaming current surface due to the presence of HA molecules. Furthermore the plateau of the $\zeta$ - potential curve for HA-coated Ti implant, shows how the coating for HA-coated Ti implant, shows how the coating properties is predominant respect the electrolyte, meaning a stable and uniform HA layer was made.
In vivo studies, in a short time model, allow evaluate the effect of the coating on healing rate at early stage. Bone ingrowth and the quality of newly formed $(\mathrm{BIC}, \%)$ bone were the parameters under investigation

\begin{tabular}{|c|c|c|c|}
\hline \multirow[t]{2}{*}{ Parameter } & \multirow{4}{*}{$\begin{array}{l}\text { Median } \\
\text { SEM } \\
\text { (Min- } \\
\text { Max) }\end{array}$} & \multicolumn{2}{|c|}{ Screw } \\
\hline & & Uncoated & Coated \\
\hline \multirow[t]{2}{*}{ BIC (\%) } & & $\begin{array}{c}22.5 \\
5.8\end{array}$ & $\begin{array}{c}69.0^{* * *} \\
5.8\end{array}$ \\
\hline & & $(16.1-47.4)$ & $(45.4-80.7)$ \\
\hline \multirow[t]{3}{*}{ Bone Ingrowth (\%) } & Median & 30.3 & $56.3^{* *}$ \\
\hline & $\begin{array}{l}\text { SEM } \\
\text { (Min- }\end{array}$ & & \\
\hline & Max) & $(23.9-33.9)$ & $(41.9-59.5)$ \\
\hline
\end{tabular}

Ha coated Ti implant significantly increases bone ingrowth at 4 weeks. Furthermore, BIC, \% significantly increase for HA-coated implant meaning that interfacial bone is more mature
compared with the Ti-implant control.

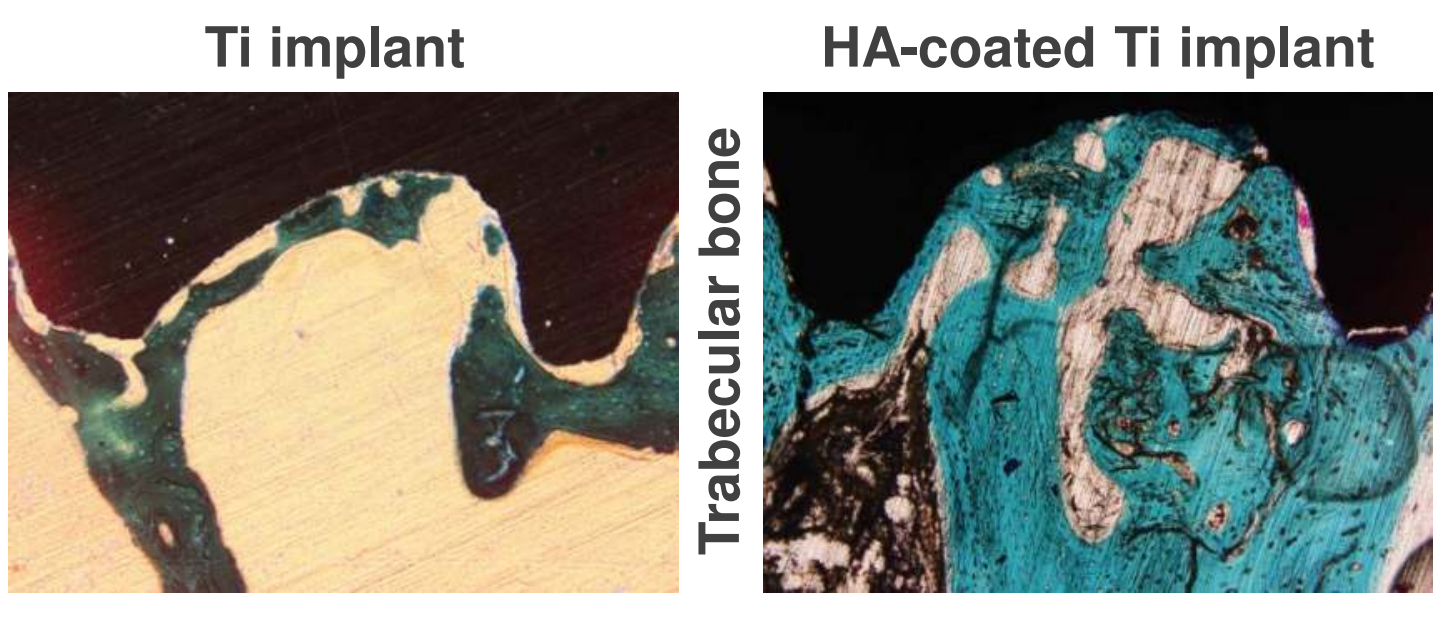

Functional studies confirm the significant improvement of osteointegration at early stage; and the histological analysis underline the increase in the biochemical markers (collagen, osteocalcin alkaline phosphatase) related to bone formation, in the trabecular bone.

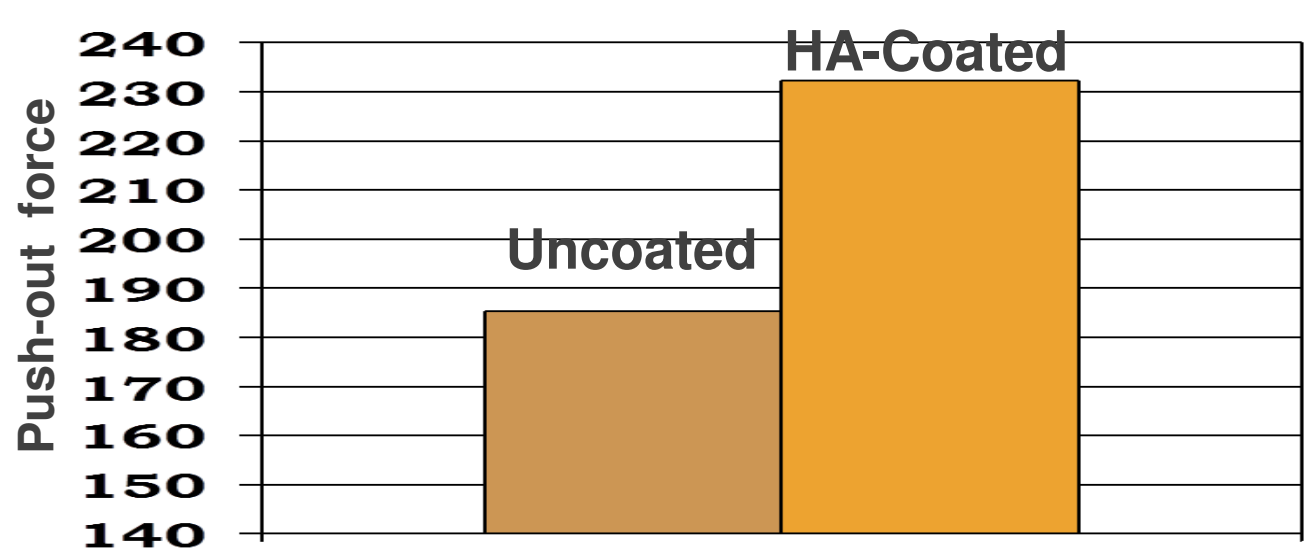

Push-out force test, was used to understand how solidly was anchored the bone to the implant. It is a measure on the side of cortical bone, and the results shows that was needed a much higher force to pushing out HA-coated Ti implant, from its bony bed than, than Ti implant uncoated.

As to the clinical test, no differences at 1 year were observed with regard to the insertion and stability between the two types of implants (test and control) during surgery in the post-operative visits with regard to the indices of inflammation and wound healing. All interventions have had an optimal healing, in the variability of the surgica situation, and no adverse events were recorded Follow up at longer time period will provide data on impact on early perimplantitis symptoms

\section{Conclusions}

In vitro results and animal tests confirm the hypothesis that turning the surface chemistry of titanium into that of hyaluronan positively affects inflammatory and bone regeneration mechanisms.

Clinical data show successful outcome and no effect on tissue resorption with respect to control at 1 year, in a cohort of healthy patients.

This set of data provides ethical foundation for further clinical tests on hyaluronan engineered implants in patients with compromised inflammatory response and ensuing evaluation of clinical indications in peri implantitis-prone patients cohorts.

\section{References}

1. M. Morra et al., J. Ortoph. Res., 27, 657, 2009

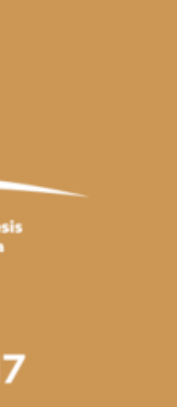

Contact:

Dr. Marco Morra mmorra@nobilbio.it
Dicencin

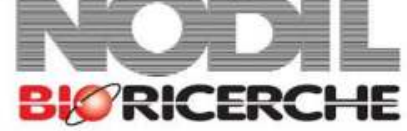

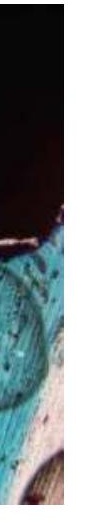

